\title{
Cation ordering and redox chemistry of layered Ni-rich NCMs: an operando Raman Spectroscopy study
}

\author{
Eibar Flores ${ }^{\mathrm{a} *}$, Petr Novák ${ }^{\mathrm{a}}$, Ulrich Aschauer ${ }^{\mathrm{b}}$, Erik J. Berga,c* \\ a: Electrochemistry Laboratory, Paul Scherrer Institute, 5232 Villigen, Switzerland \\ b: Department of Chemistry and Biochemistry, University of Bern, CH-3012 Bern, Switzerland \\ c: Department of Chemistry, Ångström Laboratory, Uppsala University, Box 538, SE-751 21 Uppsala, Sweden.
}

\begin{abstract}
Although layered transition metal oxides are the state-of-the-art cathode active materials for Li-ion batteries, many fundamental aspects of their operation are poorly understood; in particular how the local lattice structure and the transition metal composition influence their electrochemical activity. In this work, the local structure and redox activity of NCM111, NCM622 and NCM811 Li-ion cathodes are characterized under standard and overcharge operating conditions with a recently-developed operando Raman spectroscopy methodology. Supported by DFT phonon calculations and advanced data-analysis methods, we demonstrate that the Raman spectra of NCMs entail spectroscopic signatures of cation ordering phenomena, the sequential oxidation/reduction of nickel and the participation of bulk lattice oxygen in the charge-compensation process at low state of lithiation. Our methodology enables monitoring such processes during cycling and offers the potential for investigating the mechanisms by which certain strategies (i.e. doping, surface coatings, etc.) ameliorate electrochemical performance.
\end{abstract}

\section{Introduction}

Layered transition metal (M) oxides $\mathrm{LiMO}_{2}$ are currently the highest performing cathode active materials available for Li-ion batteries because of their superior combination of energy, power, lifetime, safety characteristics, and cost. Several members of this class of materials have reached the market and are widely used in portable electronics, power tools, and more recently in automotive applications. ${ }^{1,2}$ The mixed transition metal oxides with the general formula $\mathrm{LiNi}_{a} \mathrm{Co}_{b} \mathrm{Mn}_{1-\mathrm{a}-\mathrm{b}} \mathrm{O}_{2}-$ often referred to as NCMs - have been developed in order to combine the beneficial traits of each end member, i.e. the high practical specific charge of $\mathrm{LiNiO}_{2}$, the operating stability of $\mathrm{LiCoO}_{2}$ and the improved thermal stability of $\mathrm{LiMnO}_{2}{ }^{3}$ Particularly the Ni-rich NCMs have been favored in recent years and several compositions containing 33\% $\left(\mathrm{LiNi}_{0.33} \mathrm{Co}_{0.33} \mathrm{Mn}_{0.33} \mathrm{O}_{2}, \quad\right.$ abbrev. NCM111), $\quad 60 \%$ $\left(\mathrm{LiNi}_{0.6} \mathrm{Co}_{0.2} \mathrm{Mn}_{0.2} \mathrm{O}_{2}\right.$, abbrev. NCM622), and up to $80 \%$ $\left(\mathrm{LiNi}_{0.8} \mathrm{Co}_{0.1} \mathrm{Mn}_{0.1} \mathrm{O}_{2}\right.$, abbrev. NCM811) in the $\mathrm{M}$ layer have therefore been intensively explored and developed. ${ }^{4}$ However, the concentration of each $\mathrm{M}$ introduces significant differences in the physicochemical properties of these oxides and several parameters governing their electrochemical stability remain poorly understood.

Formally, the cycling of NCMs involves the topotactic de/lithiation of the lattice structure and the concomitant oxidation/reduction of the transition metals. The formal picture is, however, further modulated by the occurrence of several phenomena related to the chemical nature of $\mathrm{M}$, in particular lithium ordering and oxygen redox. Depending on $\mathrm{x}$ in $\mathrm{Li}_{\mathrm{x}} \mathrm{MO}_{2}$ the composition might separate into two phases with distinct $\mathrm{Li}$ ordered/disordered configurations. (De)lithiation through a twophase coexistence domain proceeds as a discontinuous, configurational phase transition and the electrode potential remains constant, which manifests itself as a plateau in the constant-current cycling profile. ${ }^{5-7}$ The electrochemical cycling of $\mathrm{Li}_{\mathrm{x}} \mathrm{MO}_{2}$ through thermodynamically different phases affects $\mathrm{Li}^{+}$transport ${ }^{8}$ and requires surpassing of successive energy barriers, which is believed to slow down the kinetics of the intercalation reaction. Phase transformations lead in addition to anisotropic volume changes and internal stresses that compromise the mechanical integrity of the active material particles. ${ }^{6,-11}$ Furthermore, lattice oxygen might participate in the charge-compensation process when a significant overlap between $\mathrm{O} 2 p$ states and $\mathrm{M} 3 d$ states enables charge depletion from oxygen atoms, particularly at high positive potentials. ${ }^{12}$ Oxygen redox influences the structural stability and reactivity of the active material by initiating the formation of Li-blocking reconstructed interphases ${ }^{13,14}$ that promote particle cracking ${ }^{15}$ and are accompanied by the release of reactive oxygen species. ${ }^{16}$ Several evidences of the electrochemical activity of lattice oxygen within NCM have recently been presented. ${ }^{17,18}$ Since each $M$ adapts to a unique configuration heavily influenced by the environment of its $\mathrm{M}$ neighbors, the further combination of multiple $\mathrm{M}$ in NCMs introduces additional complexity to the aforementioned cycling processes. ${ }^{19}$ Particularly the influence of each $\mathrm{M}$ on the structural stability and performance of the NCMs is intensively debated and further fundamental understanding thereof is highly desired.

Classical characterization techniques, such as x-ray and neutron diffraction, have provided the basis for our understanding of the atomic structure of NCMs. However, diffraction techniques only monitor the long-range structure of the oxides, while the electrochemistry has been shown to also be highly influenced by short-ranged phenomena, such as cation ordering. ${ }^{20}$ Because of inherent experimental limitations, many local probes study the materials ex-situ, that is, outside their working environment. Ex situ comparisons might however be misleading due to artifacts introduced by experimental preparation procedures and sample relaxation effects in which the dynamic state of the materials is lost. Therefore, the cycling-dependent evolution of the local structure of NCMs is only reliably analyzed by operando probing an individual particle within the composite electrode during operation of the Li-ion cell. ${ }^{21}$

Raman spectroscopy is a highly versatile technique widely used to study electrode materials via their periodic atomic vibrational displacements. $^{22}$ The selection rules determining the Raman activity of these vibrations are constrained by local lattice symmetry, ${ }^{23}$ so atomic environments and their distortions can 
be readily identified. In addition, the technique is ideal for operando studies of individual particles due to the optical nature of the analytical probe. The high sensitivity of conventional spectrometers enable i) the use of ordinary transparent windows for probing the active materials within their operating environment, ii) the coupling with optical microscopes for spatially resolving individual secondary particles and iii) fast recording times for time-resolved characterization of the materials during cycling. ${ }^{22}$

Despite these advantages, operando Raman measurements on $\mathrm{LiMO}_{2}$ have proven difficult due to their inherently weak Raman response, the sub-optimal electrochemical performance of the in situ cells and interfering signals from the electrolyte. In addition, the Raman active modes of many NCMs are highly overlapped so the individual contributions of the normal modes cannot be reliably deconvoluted. ${ }^{21}$ We have recently developed a custom-made operando electrochemical cell for cycling cathode materials reproducibly and simultaneously recording their Raman spectra with high spectral quality and time resolution. With the upgraded setup, we have recorded and analyzed the operando Raman spectra of $\mathrm{Li}_{\mathrm{x}} \mathrm{Ni}_{0.8} \mathrm{Co}_{0.15} \mathrm{Al}_{0.05} \mathrm{O}_{2}$ (NCA). The $x$-dependent spectral features revealed structural transformations driven by Li-ordering, previously undetected by diffraction techniques. Li-ordered domains remain shortranged as they do not modify the crystallographic lattice symmetry, but are observed as new Raman active modes that appear reversibly at low $x^{20}$

In this work, we record the operando Raman spectra of Ni-rich $\mathrm{Li}_{\mathrm{x}} \mathrm{Ni}_{1-2 \mathrm{y}} \mathrm{Co}_{\mathrm{y}} \mathrm{Mn}_{\mathrm{y}} \mathrm{O}_{2}(\mathrm{y}=0.1,0.2,0.33)$ composite electrodes with high spectral quality and time resolution. With the use of DFT calculations and automated experimental data analysis routines we perform a data-intensive interpretation of the spectra in which we combine i) spectral fitting ii) bi-dimensional correlation and iii) multivariate curve resolution analysis. We aim to elucidate the local structural evolution of NCMs during dis-/charge cycling.

\section{Materials and methods}

Electrode Preparation. The NCM composite electrodes were prepared from mixed slurries of $89 \mathrm{wt} \%$ active material (NCM powders, stored in an Ar-filled glovebox, received from BASF SE), 5 wt \% polyvinylidene difluoride (PVdF Kynar HSV 9000, Arkema), 4.6 wt \% amorphous carbon Super C65, and 1.4 wt \% graphite SFG6 (Imerys Graphite and Carbons) dispersed in n-methyl pyrrolidone solvent (NMP, SigmaAldrich). The slurries were coated onto Celgard 2400 (Celgard LLC) sheets by doctor blading at a $100-\mu \mathrm{m}$ wet thickness. The coated sheets were dried for $10 \mathrm{~h}$ under dynamic vacuum at 80 ${ }^{\circ} \mathrm{C}$, punched to $14-\mathrm{mm}$ diameter electrodes, further dried under dynamic vacuum at $80^{\circ} \mathrm{C}$ overnight, and finally introduced into an argon filled glovebox without air exposure.

Raman Spectro-Electrochemical Cell Assembly. The operando Raman measurements were performed using a custom-made Raman spectro-electrochemical cell, described in detail in references ${ }^{20,21}$. The cell was assembled in a coin-cell configuration inside an argon-filled glovebox $\left(\mathrm{O}_{2}, \mathrm{H}_{2} \mathrm{O}<1 \mathrm{ppm}\right)$. Before assembly, the composite electrodes and the Celgard 2400 separator $(\varnothing 17 \mathrm{~mm})$ were wetted for several minutes in LC30 electrolyte $\left(1.0 \mathrm{M} \mathrm{\textrm {LiClO } _ { 4 }}\right.$ in $1: 1 \quad(\mathrm{w} / \mathrm{w})$ ethylene carbonate (EC)/dimethyl carbonate (DMC)). The NCM working electrodes were pressed against an aluminum mesh (Ø17 mm, $5 \mathrm{Al}$ 7-125, Dexmet corporation, CT, US) for electric contact. Lithium metal disks $(0.2 \mathrm{~mm}$ thick, $\varnothing 12 \mathrm{~mm}$, Sigma-Aldrich, Germany) were used as counter electrode. The cell was cycled galvanostatically at room temperature with a computer-controlled galvanostat (CCCC Hardware, Astrol Electronic, Switzerland) at an applied current of $\sim 10 \mathrm{~mA} / \mathrm{g}_{\mathrm{NCM}}$ normalized to the weight of the NCM active material in the electrode. The cycling conditions were set to a 3.0-4.3 V window for standard cycles and a 3.0-4.8 $\mathrm{V}$ for the overcharge cycle.

Raman Spectroscopy. The Raman spectra were acquired using a Labram HR800 Raman microscope (Horiba-Jobin Yvon) with a $\mathrm{He}-\mathrm{Ne}$ excitation laser $(632.8 \mathrm{~nm})$. A grating was used as dispersion element with a groove density of $600 \mathrm{~g} / \mathrm{mm}$ that attains a $2 \mathrm{~cm}^{-1}$ spectral resolution. The hole and slit of the confocal system were fixed at 1000 and $100 \mu \mathrm{m}$, respectively. The laser was focused on the sample using a $50 \times$ (numerical aperture 0.55 ) objective, which produced a laser spot of ca. 4 $\mu \mathrm{m}$ diameter with an estimated sampling depth of around 2 $\mu \mathrm{m} .{ }^{24}$ However, opaque samples such as $\mathrm{Li}_{\mathrm{x}} \mathrm{MO}_{2}$ oxides strongly damp the laser intensity profile in accordance with the dielectric properties of the sample, thus the sampling depth is theoretically estimated to be around $160 \mathrm{~nm}$ in our case. ${ }^{25}$ The nominal laser power was filtered down below $2 \mathrm{~mW}$ to avoid sample overheating. The probed sample spot was monitored before and after the experiment to verify that it remained in focus. Every recorded spectrum resulted from the average of minimum 5 acquisitions exposed for $100 \mathrm{~s}$ each, but these parameters were increased for every material aiming at reducing spectral noise and maximizing signal intensity.

Data Analysis. For fitting, the data treatment was a two-step process as follows: first a custom-made Matlab graphical user interface (GUI) was used to visually evaluate several sample spectra in search of optimum fitting parameters such as the baseline polynomial degree, number of peaks, and the initial guesses and boundaries of peak position and width of the Lorentz-type deconvolution profiles. Second, the optimum fitting parameters were input in a script to treat and fit each spectrum consistently in order to retrieve band position, intensity, and width of all Lorentz-type peaks. The correlation analysis was performed on the baseline-subtracted spectra using also a custom-made Matlab script implementing the mathematical formalism of the generalized two-dimensional correlation analysis in references ${ }^{26,27}$. The multivariate curve resolution analysis was performed using the open-source Matlab GUI and protocol provided by reference ${ }^{28}$. The state of lithiation $x$ was calculated from the cycling data file as the fraction of the specific charge at a given time relative to the theoretical specific charge of each NCM:

$$
x\left(\text { in } \mathrm{Li}_{\mathrm{x}} \mathrm{Ni}_{1-2 \mathrm{y}} \mathrm{Co}_{\mathrm{y}} \mathrm{Mn}_{\mathrm{y}} \mathrm{O}_{2}\right)=\frac{I t}{m_{\text {active }} Q_{\text {theor }}}
$$

where $I(\mathrm{~mA})$ is the galvanostatic current, $t(\mathrm{~h})$ is the elapsed cycling time, $m_{\text {active }}(\mathrm{g})$ is the mass of active material in the composite electrode and $\mathrm{Q}_{\text {theor }}$ the theoretical specific charge of the particular NCM.

Computational methods. The density functional theory (DFT) calculations were carried out using the VASP $\operatorname{code}^{29-31}$, using the Perdew-Burke-Ernzerhof exchange correlation functional. ${ }^{32}$ Electron-core interactions were described by PAW potentials $^{33,34}$ with $\mathrm{Li}(1 s, 2 s), \mathrm{Ni}(4 s, 3 p, 3 d), \mathrm{Co}(4 s, 3 p, 3 d)$, $\mathrm{Mn}(4 s, 3 p, 3 d)$ and $\mathrm{O}(2 s, 2 p)$ valence electrons and wavefunctions were expanded in planewaves up to a cutoff energy of $500 \mathrm{eV}$. We applied a Hubbard $U^{35,36}$ to the transition metal $d$ states with $U_{\mathrm{Ni}}=6.70 \mathrm{eV}, U_{\mathrm{Co}}=4.91 \mathrm{eV}$ and $U_{\mathrm{Mn}}=$ $4.89 \mathrm{eV}^{37}$ The calculations were carried out with an antiferromagnetic order with up spins on $\mathrm{Ni}$ and down spins on Mn. NCM 111 was modeled as a 108 atom hexagonal cell with $\mathrm{a}=\mathrm{b}=8.68 \AA$ and $\mathrm{c}=14.35 \AA$, for which reciprocal space was sampled with a $\Gamma$-centered $2 \times 2 \times 1$ mesh. All internal and cell degrees of freedom were relaxed until forces converged below $10^{-3} \mathrm{eV} / \AA$ and stresses below $5 \cdot 10^{-3} \mathrm{eV} / \AA^{3}$. Phonon frequencies were computed using the frozen phonon method as implemented in the phonopy package. ${ }^{38}$ The mode intensities were computed from the mode-dependent change in dielectric 
constant ${ }^{39}$ evaluated via finite differences for mode amplitudes of $\pm 0.01 \AA$ and dielectric constants computed using density

\section{Results and discussion}

\section{Electrochemistry}

Figure 1 shows the galvanostatic potential profiles of the NCM111, NCM622 and NCM811 based composite electrodes during the $2^{\text {nd }}$ cycle up to $4.3 \mathrm{~V}$. The $2^{\text {nd }}$ cycle is more representative of the reversible electrochemical reactions of the oxides as most activation processes end in the first cycle. ${ }^{40}$ The electrochemistry is in general agreement with numerous previous reports on these intensively investigated class of NCM oxides, ${ }^{41-44}$ thus validating the electrochemical performance of the Raman spectro-electrochemical Li-cell. A higher Ni content expectedly increases the available specific charge $(157 \mathrm{mAh} / \mathrm{g}$ for NCM111, $176 \mathrm{mAh} / \mathrm{g}$ for NCM622, and $203 \mathrm{mAh} / \mathrm{g}$ for NCM811 up to $4.3 \mathrm{~V}$ ) as $\mathrm{Ni}$ is the main redox active chargecompensating specie. ${ }^{45-47}$ The activation processes during the first cycle cause an initial irreversible charge loss $(23 \mathrm{mAh} / \mathrm{g}$ for $\mathrm{NCM} 111,16 \mathrm{mAh} / \mathrm{g}$ for NCM622, and $32 \mathrm{mAh} / \mathrm{g}$ for NCM811 between 3.0 and $4.3 \mathrm{~V}$ ) likely associated with the dissolution of surface impurities (e.g. Li-/ $\mathrm{NiCO}_{3}$ and/or $\mathrm{LiOH})^{41,48}$ and surface reconstruction reactions (e.g. $\mathrm{O}_{2}$ release).$^{17}$ Overpotentials increase on overcharge ( $3^{\text {rd }}$ cycle, Figure S1) as a consequence of degradation phenomena including electrolyte oxidation, ${ }^{49}$ microstructural deterioration, ${ }^{9}$ transition metal dissolution, ${ }^{50}$ enhanced cation disorder $^{8}$ and surface reconstruction. ${ }^{14}$

The plateau-like regions in the potential profile (represented by peaks in the differential charge curves, Figure 1) indicate the occurrence of phase separation, ${ }^{7}$ generally associated with $\mathrm{Li}^{+}$ order/disorder (configurational) transitions during $\mathrm{NCM}$ (de)lithiation. ${ }^{6,51}$ X-ray diffraction studies of $\mathrm{Li}_{x} \mathrm{NiO}_{2}$ have shown that such transitions also drive structural changes between hexagonal and monoclinc phases. ${ }^{52,53}$ Nevertheless, all three NCMs (de)lithiate following a solid solution mechanism, i.e., only one crystallographic phase is observed by diffraction techniques during electrochemical cycling. ${ }^{42,54}$ Using Raman spectroscopy we have recently demonstrated that the same transformations between Li-ordered phases also occur in the mixed transition metal oxide NCA, but remain short-ranged since no crystallographic phase separation occurs. ${ }^{20}$ Some of the electrochemical features observed in Figure 1 might also result from transitions between locally ordered Li domains. Particularly the redox peak at $4.2 \mathrm{~V}$ observed in Ni-rich NCMs is of interest, since it coincides with an abrupt contraction of the rhombohedral lattice along the c-axis ${ }^{43}$ and correlates with a series of degradation processes leading to rapid capacity fading during cycling. ${ }^{55}$ Raman spectroscopy is hence pursued in order to gain further insights into local structural processes possibly having a deleterious influence on the cycling process and reversibility of the composite electrodes. functional perturbation theory.

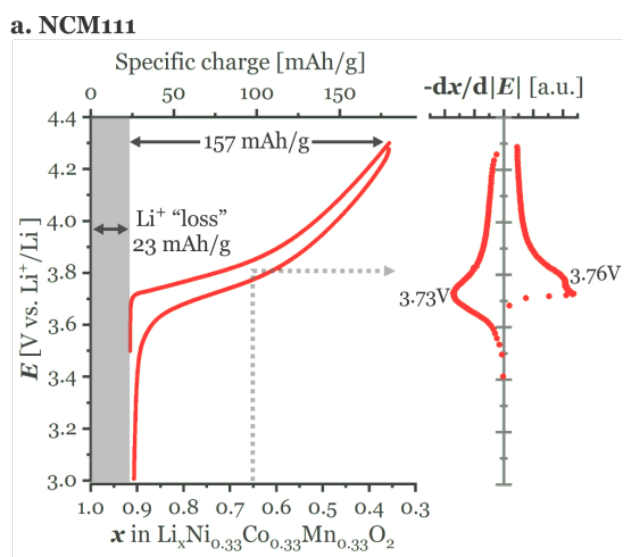

b. NCM622
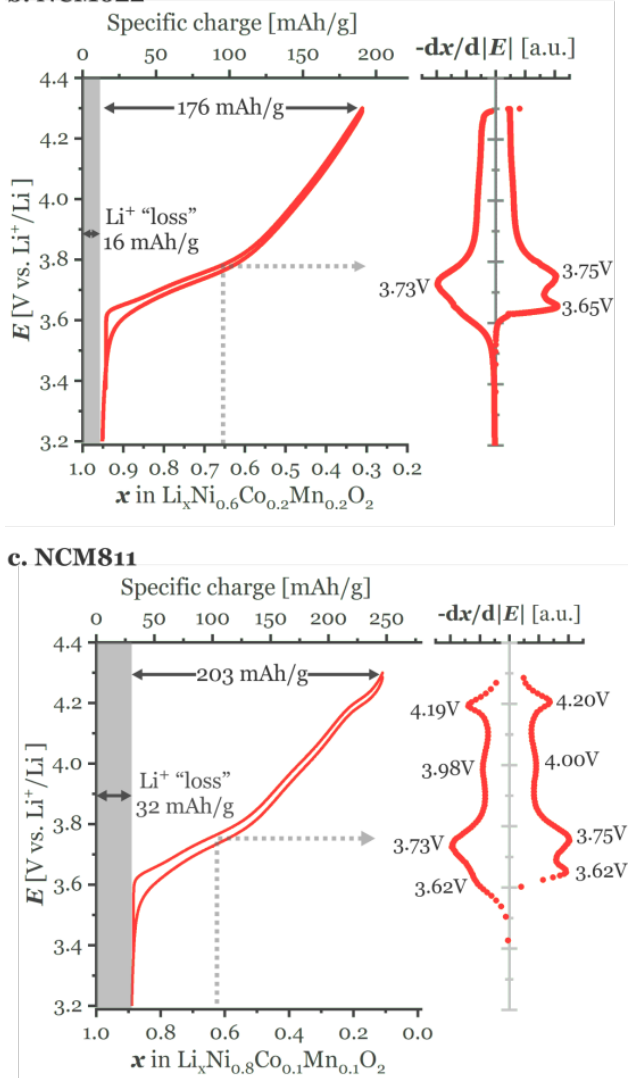

Figure 1. Galvanostatic potential profiles and differential charge curves of the a. NCM111, b. NCM622 and c. NCM811 based composite electrodes during the second cycle $\left(3.0-4.3 \mathrm{~V}\right.$ vs. $\mathrm{Li}^{+} / \mathrm{Li}$, $10 \mathrm{~mA} / \mathrm{g}$ rate), cycled in the spectro-electrochemical Raman cell. The arrows indicate the SOL value where the redox peak ca. 3.74 $\mathrm{V}$ takes place.

Raman Spectra of lithiated NCM

Most NCMs share the same rombohedral, layered lattice structure belonging to the R-3m space group and can be described as a cubic close-packed oxygen sublattice in which $\mathrm{Li}$ and the transition metals $M$ arrange in octahedral sites forming alternated layers (Figure S3a). ${ }^{41}$ Assuming that all ion site symmetries are crystallographically refined from the R-3m space group, namely, $\mathrm{Li}$ in $3 \mathrm{a}$ Wyckoff sites $(-3 m$ site symmetry or $D_{3 d}$ in Shoenflies notation $\left.{ }^{56}\right), \mathrm{M}$ in $3 \mathrm{~b}$ sites $(-3 m$ or $\left.D_{3 d}\right)$ and $\mathrm{O}$ in $6 \mathrm{c}$ sites $\left(3 m\right.$ or $\left.C_{3 v}\right),{ }^{57}$ a factor group analysis predicts two Raman active modes: the $\mathrm{E}_{\mathrm{g}}$ mode involving opposing oxygen displacements along adjacent O-layers and the $A_{1 g}$ mode where oxygens move symmetrically along the caxis (sup info S3b). ${ }^{58}$ These two Raman active modes hence result from oxygen vibrations only and the spectral trends reflect primarily the state and evolution of the local oxygen 
environment. Both computational models and experimental studies of $\mathrm{LiCoO}_{2}$ validate such an assumption. ${ }^{20}$ However, when Co is partly substituted for other transition metals (e.g. $\mathrm{Ni}, \mathrm{Mn})$ a more heterogeneous oxygen environment results ${ }^{19}$ and the Raman spectrum is expected to reflect a distorted $\mathrm{C}_{3 \mathrm{v}}$ site symmetry (Figure S4). Indeed, compared to our previous DFT calculations on $\mathrm{LiCoO}_{2},{ }^{20}$ the fully lithiated NCM111 show significantly broader spectral envelopes (Figure 2a) resulting from more complex oxygen vibrational motions than derived from factor group analysis of the R-3m space group. ${ }^{59}$ For instance, the highest intensity peak at $566 \mathrm{~cm}^{-1}$ involves oxygen displacement vectors pointing in multiple directions, and can neither be fully identified as an $\mathrm{E}_{\mathrm{g}}$ nor $\mathrm{A}_{1 \mathrm{~g}}$ mode (Supp info S3b). In fact, all modes with non-negligible Raman activity feature complex vibrational patterns, the most intense ones being illustrated in supplementary information movies. Note however that the eigenvectors of the most intense $566 \mathrm{~cm}^{-1}$ mode (Figure 2b) are antisymmetric (i.e. point in opposing directions) with respect to $\mathrm{M}$ and $\mathrm{Li}$ planes, suggesting that some symmetry properties are still preserved. Hence, by comparing the computed patterns of NCMs with those from an ideal R-3m lattice (Figure S3b), some modes can at best be said to be $\mathrm{A}_{\mathrm{g}}$-like or $\mathrm{E}_{\mathrm{g}}$-like, but most modes feature complex eigenvectors resembling none of these two modes. In any case, only oxygen displacements are able to modify the lattice's polarizability, thus rendering their vibrations Raman-active (Figure S3c), and the Raman spectra of all NCMs respond primarily to oxygen's interactions with its cationic ( $\mathrm{Li}$ and transition metal) neighbors.

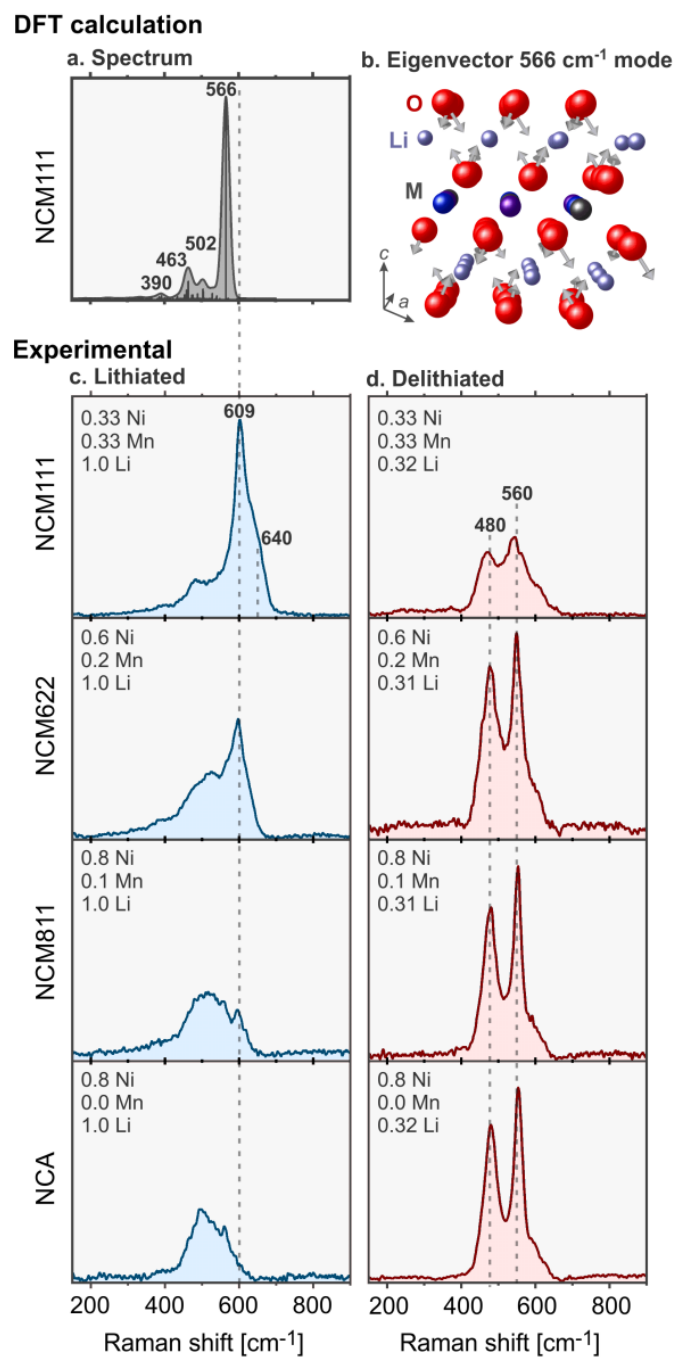

Figure 2. a. DFT-calculated Raman spectrum of NCM111 and b. the eigenvectors of the mode at $566 \mathrm{~cm}^{-1}$. Ex-situ Raman spectra of c. lithiated and d. partially delithiated NCM powders. The delithiated oxides have been electrochemically charged up to 190 $\mathrm{mAh} / \mathrm{g}$ which correspond to $\mathrm{ca}$. 2/3 Li removed per formula unit.

Figure 2c shows the experimental Raman spectra of fully lithiated NCM111, NCM622, and NCM811 materials along with NCA (Mn-free) for comparison. All spectra are consistent with previous reports ${ }^{60-62}$, except for NCM622 which is here reported for the first time. The broad spectral envelope measured for NCM111 and its qualitative agreement with the DFT data suggest that the mix of transition metals indeed introduce distortions to the oxygen local environment in the crystal structure of the as-synthesized material.

In the calculated NCM111 structure, all Ni, Co and $\mathrm{Mn}$ are found in 2+, 3+(low spin) and 4+ oxidation states, respectively (Figure S5a), agreeing well with previous calculations ${ }^{63}$ and $\mathrm{X}$ ray absorption spectra ${ }^{45,64,65}$. In addition, no Jahn-Teller distorted M's are found according to the quantification of the Q3 displacement mode (Figure S5b) ${ }^{66}$ Hence, these distortions do not originate from electronic degeneracy but might rather result from i) the strain of accommodating edge-sharing $\mathrm{MO}_{6}$ octahedra with inherently different volumes ${ }^{19}$ and/or ii) magnetic exchange interactions between M's. ${ }^{67}$ Interestingly, we found that in the lowest energy NCM111 structure no M repeats in the first coordination shell of an oxygen atom. That is only realized if M's order in a repeating pattern of Ni-Co-Mn triangles (Figure S5c). As mentioned previously, this calculated LE structure is the only one featuring a Raman spectrum that compares well to the experimental one. Consequently, we believe that M's maintain a certain degree of ordering in the assynthesized material. In support, $\mathrm{M}$ ordering should result in a $[\sqrt{ } 3 \times \sqrt{ } 3] \mathrm{R} 30^{\circ}$ superlattice, ${ }^{68}$ which has been experimentally observed by other local probes. ${ }^{65,69}$ However, the ordering apparently remains short-ranged since diffraction techniques have not detected any superlattice features. ${ }^{54,70,71}$

While comparing the spectra of the litihated $\mathrm{LiMO}_{2}$ oxides in Figure 2c, it is obvious that all lithiated Mn-containing oxides show a band centered ca. $600 \mathrm{~cm}^{-1}$, the intensity of which scales with the Mn content. The band is absent in Mn-free NCA so it clearly results from Mn substitution. ${ }^{21}$ However, at this point it cannot be ascribed exclusively to Mn-related environments, since the fraction of both $\mathrm{Mn}^{4+}$ and $\mathrm{Ni}^{2+}$ equally increase according to $\mathrm{Li}\left[\mathrm{Ni}^{2+}\right]_{\mathrm{a}}\left[\mathrm{Ni}^{3+}\right]_{1-2 \mathrm{a}-\mathrm{b}}\left[\mathrm{Co}^{3+}\right]_{\mathrm{b}}\left[\mathrm{Mn}^{4+}\right]_{\mathrm{a}} \mathrm{O}_{2}$ with higher $\mathrm{Mn}$ content. Figure $2 \mathrm{c}$ also shows that the band features a shoulder at higher wavenumbers (c.a. $640 \mathrm{~cm}^{-1}$ ). DFT calculations (Figure S5d) suggest this shoulder might result from either $\mathrm{M}$ disorder or $\mathrm{M} / \mathrm{Li}$ interlayer exchange. $\mathrm{Ni}^{2+}$ is well-known to migrate to lithium sites left vacant after synthesis or delithiation, ${ }^{72}$ since both cations feature comparable Shannon radii $\left(\mathrm{r}_{\mathrm{S}}\left[\mathrm{Ni}^{2+}\right]=69 \mathrm{pm}, \mathrm{r}_{\mathrm{S}}\left[\mathrm{Li}^{+}\right]=76 \mathrm{pm}\right){ }^{73}$

\section{Raman spectra of delithiated NCM}

Figure $2 \mathrm{~d}$ (right) shows that the spectra for the various $\mathrm{Li}_{0.3} \mathrm{MO}_{2}$ compositions are qualitatively similar featuring a pronounced pair of bands at $480 \mathrm{~cm}^{-1}$ and $560 \mathrm{~cm}^{-1}$, where the $E_{\mathrm{g}}$ and $A_{1 \mathrm{~g}}$ modes characteristic of the ideal $\mathrm{R}-3 \mathrm{~m}$ lattice are expected to appear. ${ }^{20}$ Hence, we believe that oxygen environments in the M-distorted lattice approach the ideal $3 m$ site symmetry upon lithium extraction. These bands become increasingly intense for NCMs of higher $\mathrm{Ni}^{4+}$ content. Based on DFT calculations, we previously proposed that the Raman intensities of oxygen vibrations in $\mathrm{LiMO}_{2}$ increase with increasing M-O covalency. ${ }^{20}$ The Raman spectra in Fig. 1d and other DFT calculations ${ }^{74}$ support the hypothesis that intense $\mathrm{E}_{\mathrm{g}} / \mathrm{A}_{1 \mathrm{~g}}$ bands relate to highly covalent $\mathrm{Ni}^{4+}-\mathrm{O}$ bonds. Our DFT calculations suggest that the shoulders $>600 \mathrm{~cm}^{-1}$ originate from Li-vacancy formation, which although weak affects the spectra for all delithiated NCMs. The differences between the lithiated and delithiated spectral profiles of the oxides indicate that the Raman-active 
modes are strongly influenced by the concomitant effects of charge compensation and lithium (de)insertion on the oxygen local environment.

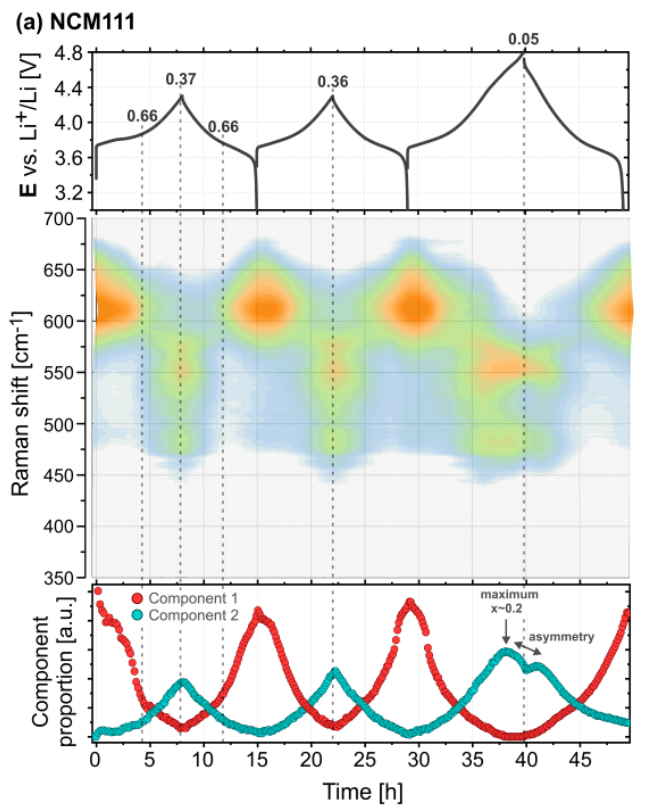

(b) NCM622
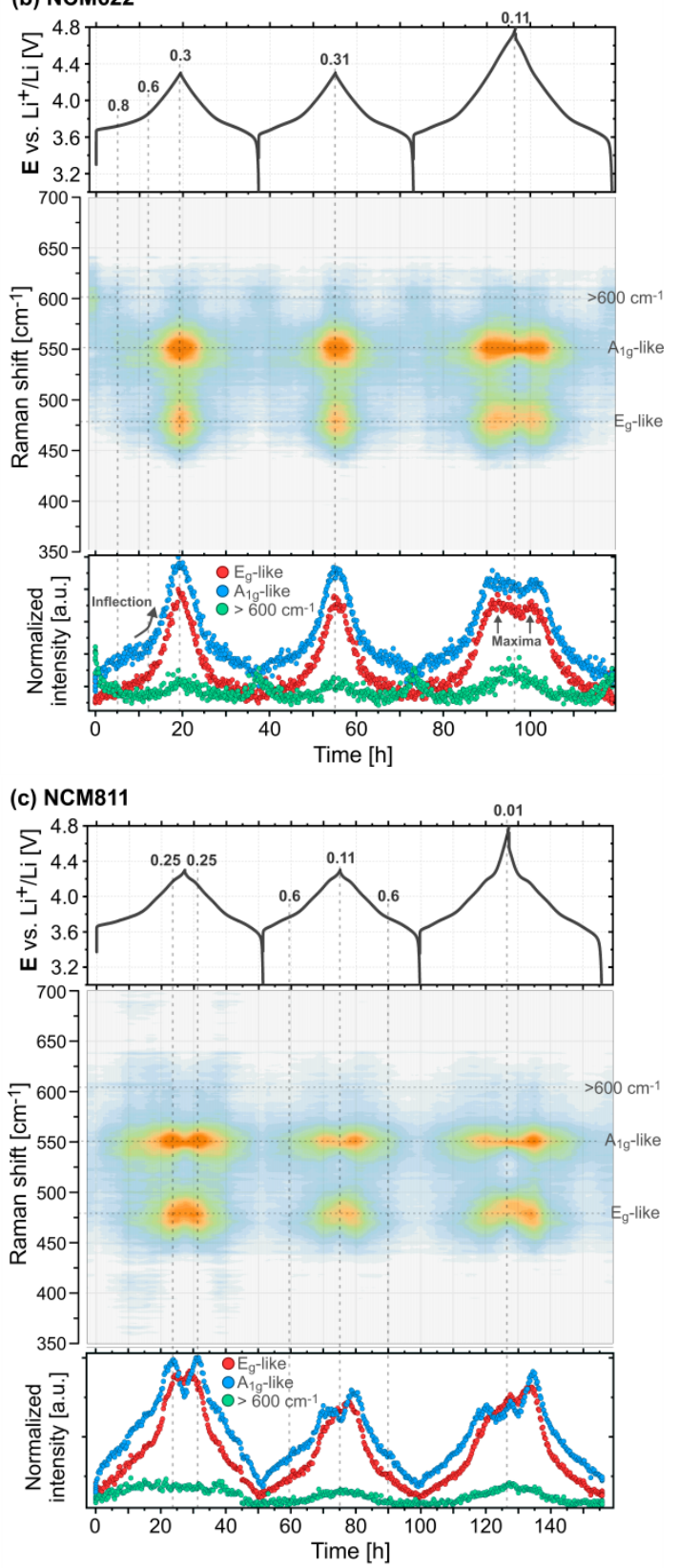

Figure 3. Potential-dependent evolution of Raman spectra of (a) NCM111, (b) NCM622, and (c) NCM811 along with the component proportions/fitted peak intensities.

\section{Raman spectra evolution during cycling}

Figure 3 shows the potential profiles and the contour maps representing the evolution of the Raman spectra of NCM111, NCM622 and NCM811 during three constant-current cycles. The spectral evolution is progressive and reversible between the two extremes represented by spectra of the lithiated and delithiated compositions (Figure 2). Apart from the general similarity among the spectra of $\mathrm{Li}_{0.3} \mathrm{MO}_{2}$, the pair of bands at $\sim 480$ and $\sim 560 \mathrm{~cm}^{-1}$ evolve similarly upon cycling in terms of peak position and intensity trends, thus evidencing that they all share the same oxygen local environment modulated by $\mathrm{Ni}^{4+}-\mathrm{O}$. However, the bands centered around $600 \mathrm{~cm}^{-1}$ behave differently in the three oxides; more specifically, in NCM111 the band is the strongest in the lithiated state, while in NCM811 the band reaches maximum intensity close to the end of delithiation. In order to visualize more clearly the contrasting behaviors of the band centered around $600 \mathrm{~cm}^{-1}$ a generalized correlation analysis of the time-dependent spectra can be performed (Figure S6). The band at around $600 \mathrm{~cm}^{-1}$ correlates to the $(550,475) \mathrm{cm}^{-1}$ pair negatively in NCM111, but positively in NCM811, indicating they originate from fundamentally different vibrations. Likely, the bands at around $600 \mathrm{~cm}^{-1}$ in the lithiated oxides are introduced by $\mathrm{Mn}$ substitution (Figure 2b) whereas Li-vacancies explain the band around $600 \mathrm{~cm}^{-1}$ appearing during delithation. ${ }^{20}$ In NCM622, the comparable but contrasting correlations cancel out, resulting in dim cross-correlation zones. Mn-related bands do not necessarily occur at the same frequency as Li-vacancy bands but given their wide profiles they cannot be deconvoluted.

\section{Spectral behavior of NCM111}

For $\mathrm{Li}_{\mathrm{x}}\left(\mathrm{Ni}_{1 / 3} \mathrm{Co}_{1 / 3} \mathrm{Mn}_{1 / 3}\right) \mathrm{O}_{2}$, the $x$-dependent spectra can be divided into two main stages above and below $x \sim 0.66$ (dashed line in Figure 3a): an initial stage characterized by the weakening of the band centered at $609 \mathrm{~cm}^{-1}$ and a later stage displaying the appearance and growth of a band triplet at 480 , 555 , and $575 \mathrm{~cm}^{-1}$ (detailed in Figure S7a). The stages span the expected oxidation intervals characteristic of the two Ni redox couples: in the interval $1.0>x>0.66$ the governing redox process is $0.33 \mathrm{Ni}^{2+} \rightarrow 0.33 \mathrm{Ni}^{3+}$ and in $0.66>x>0.33$ the governing redox process is $0.33 \mathrm{Ni}^{3+} \rightarrow 0.33 \mathrm{Ni}^{4+}$, thus charge-compensating for the removal of $0.66 \mathrm{Li}^{+}$in total. ${ }^{45}$ Therefore, we conclude that the strong Raman band at $609 \mathrm{~cm}^{-1}$ is likely characteristic of $\mathrm{Ni}^{2+}-\mathrm{O}$ environments introduced by $\mathrm{Mn}$ substitution, as its intensity drops where the oxidation of $\mathrm{Ni}^{2+}$ to $\mathrm{Ni}^{3+}$ is expected to occur. Likewise, the $\mathrm{Ni}^{4+}-\mathrm{O}$ environment is characterized by the band triplet at 480,555 , and $575 \mathrm{~cm}^{-1}$. Note that $\mathrm{Ni}^{4+}-\mathrm{O}$ related bands only appear after the disappearance of $\mathrm{Ni}^{2+}-\mathrm{O}$ bands (Figure 3a), confirming experimentally that nickel oxidation is a two-step process: $\mathrm{Ni}^{2+} / \mathrm{Ni}^{3+}<_{-}>\mathrm{Ni}^{3+} / \mathrm{Ni}^{4+}$. ${ }^{45}$ The overall spectrum is weakest when the $\mathrm{Ni}^{3+}-\mathrm{O}$ environments are expected to be dominant, probably as a result of the disorder introduced by a non-cooperative Jahn-Teller distortion due to the low-spin $\mathrm{Ni}^{3+}$ environment. ${ }^{75}$

Further details about the spectral evolution can be revealed if each spectrum is fitted and the fitting parameters are studied versus lithium content. However, given the highly overlapping nature of the bands for NCM111, there are multiple fitting solutions for each spectrum; hence, an automated fitting procedure would unavoidably involve arbitrary parameters not representative of the physical system. Instead of attempting a deconvolution into Lorentz-type profiles, we have applied a multivariate curve resolution (MCR-ALS) analysis, which decomposes the time-dependent spectra into a linear combination of "pure" components, each weighted according to 
their relative abundance. ${ }^{28}$ We found that the resulting two first components and their proportions are appropriate representations of the $x$-dependent fractions of lithiated and delithiated NCM111 (Figure S7b). Naturally, component 1 fades at the expense of the growth of component 2 during delithiation (Figure 3a), and the trends are reversible upon lithiation. However, on overcharge the trends are asymmetric with respect to the end of charge (hysteresis in the SOL representation of Figure S7c), likely reflecting irreversible structural degradations. Component 2 inflects near $x=0.2$, but no relation to configurational transitions between $\mathrm{Li}$-ordered phases are expected, ${ }^{51}$ since at these low SOL there is neither electrochemical (Figure S1) nor crystallographic evidence of phase separation. ${ }^{42,43}$ Given that the Raman intensities increase with Ni-O covalency ${ }^{20}$ the inflection would thus represent a depletion of electrons from the same bond. Indeed, $\mathrm{CO}_{2} / \mathrm{O}_{2}$ evolution associated to lattice oxygen release from NCM111 has been observed at the same SOL, ${ }^{17}$ thus implying that electron depletion from the $\mathrm{Ni}^{4+}-\mathrm{O}$ bond and oxygen release are intimately linked also for the NCM class of materials.

\section{Spectral behavior of NCM622}

Figure $3 b$ shows the evolution of Raman spectra of NCM622 along with the corresponding constant-current profile. The spectral profile in the lithiated state is characterized by a strong band at $600 \mathrm{~cm}^{-1}$ while the spectrum of the delithiated state is dominated by the intense $\mathrm{E}_{\mathrm{g}}$ and $\mathrm{A}_{\mathrm{gg}}$-like bands at 480 and 550 $\mathrm{cm}^{-1}$ (Figure 2b). Unlike for NCM111, the bands are here sufficiently defined to confidently perform an automated fitting procedure on all spectra (Figure S8b) and the peak intensities are shown in Figure $3 \mathrm{~b}$. The peak $>600 \mathrm{~cm}^{-1}$ feature a U-shape trend that reaches minimum between $\mathrm{x}=0.8$ and $\mathrm{x}=0.6$. As discussed for NCM111, the band around $600 \mathrm{~cm}^{-1}$ in lithiated NCMs likely corresponds to $\mathrm{Ni}^{2+}$ in the structure and its disappearance at $x=0.8$ suggests the complete oxidation of $\mathrm{Ni}^{2+}$ to $\mathrm{Ni}^{3+}$. Indeed, since NCM622 is formally described as $\mathrm{Li}\left[\mathrm{Ni}^{2+}\right]_{0.2}\left[\mathrm{Ni}^{3+}\right]_{0.4}\left[\mathrm{Co}^{3+}\right]_{0.2}\left[\mathrm{Mn}^{4+}\right]_{0.2} \mathrm{O}_{2}$ due to electron transfer of $\mathrm{Mn}$ to $\mathrm{Ni}^{64}$ all $\mathrm{Ni}^{2+}$ should transform to $\mathrm{Ni}^{3+}$ after $x=0.8$. On further delithiation, the band intensity increases again shortly after the redox peak around 3.74 $\mathrm{V}$ (Figure 1b), indicating the occurrence of a configurational phase transition. ${ }^{20}$ The $\mathrm{E}_{\mathrm{g}}$ and $\mathrm{A}_{1 \mathrm{~g}}$-like peaks display a slow, linear intensity increase that accelerates after the inflection at $x \sim 0.6$ as $\mathrm{Ni}^{3+}$ is oxidized to $\mathrm{Ni}^{4+}$. During relithiation the bands return reversibly to their initial spectral profile, and the same band evolution repeats in the $2^{\text {nd }}$ cycle. However, on overcharge in the $3^{\text {rd }}$ cycle the intensities of both peaks saturate (see also Figure S8c): the $\mathrm{E}_{\mathrm{g}}$ like peak at $\mathrm{x}=0.3$ and $\mathrm{A}_{1 \mathrm{~g}}$-like peak slightly earlier $(x=0.35)$. Although no electrochemical features are found around $x=0.3$ (Figure 1b), lattice oxygen release has been observed shortly thereafter at $x=0.25 .{ }^{17}$ The intensity variations at low SOL seem also related to severe electron depletion from oxygen as in NCM111.

\section{Spectral behavior of NCM811}

Figure 3c shows that the Raman spectra of NCM811 follow the same general trend as for NCM111 and NCM622 during cycling. The band at $600 \mathrm{~cm}^{-1}$ is however hardly discernable in the fully lithiated composition $\mathrm{Li}\left[\mathrm{Ni}^{2+}\right]_{0.1}\left[\mathrm{Ni}^{3+}\right]_{0.7}\left[\mathrm{Co}^{3+}\right]_{0.1}\left[\mathrm{Mn}^{4+}\right]_{0.1} \mathrm{O}_{2}$, due to the dominance of $\mathrm{Ni}^{3+}$ and the low fraction of $\mathrm{Ni}^{2+}$. On delithiation, the pair of $\mathrm{Eg}_{\mathrm{g}}$ like and $A_{1 \mathrm{~g}}$-like bands centered at 470 and $550 \mathrm{~cm}^{-1}$ becomes more defined and rapidly intensify as $\mathrm{Ni}^{4+}$ forms and reaches maxima at $x \sim 0.20$ and 0.25 , respectively. As for NCM111 and NCM622, the fitted intensity trends in NCM811 reach maxima at the SOL where $\mathrm{CO}_{2} / \mathrm{O}_{2}$ gases are released from the active material, ${ }^{16,17}$ confirming that the maxima are spectral signatures of oxygen oxidation. Given the high SOL-resolution of the intensity trends, the inflection can be correlated with the onset of $\mathrm{CO}_{2} / \mathrm{O}_{2}$ release but is observed to occur at a lower electrode potential than the redox peak around $4.19 \mathrm{~V}$ (Figure S9). The latter has rather been correlated to the rapid $c$-axis contraction of the lattice associated to mechanical degradation of the active material. ${ }^{40}$ Oxygen redox starts before the lattice contracts abruptly, suggesting that these are two separate processes but not discarding a causality relationship between the two.

The intensity trends of the band at $\sim 600 \mathrm{~cm}^{-1}$ are noisy during the first cycle but become more defined during the second cycle where the bands clearly grow with delithiation. The bands grow and define for lithium contents below $x=0.6$, where the redox peak around $3.74 \mathrm{~V}$ is observed in Figure 1c. As for NCA, the Raman bands above $600 \mathrm{~cm}^{-1}$ are new modes introduced by the symmetry modification that Li-ordered domains introduce in the oxygen environment. ${ }^{20}$

\section{Conclusions}

The local structure and redox activity of NCM-based Li-ion cathodes was characterized by operando Raman spectroscopy complemented with DFT phonon calculations. Despite sharing the same crystallographic (long-ranged) structure, the local oxygen environments and electrochemical activity of NCMs differ considerably and are highly dependent on the particular Ni:Co:Mn proportion. By comparing the DFT-calculated and measured Raman spectra of lithiated NCM111, we show that transition metal ordering as predicted by other DFT-based studies indeed exist in the as-synthesized material but remains short-ranged as it cannot be detected by diffraction techniques.

The amount of $\mathrm{Ni}^{2+}-\mathrm{O}$ environments in the structure can be correlated with the Raman band around $610 \mathrm{~cm}^{-1}$ common for all Mn-containing oxides. The disappearance of this band upon delithiation of NCM111 and NCM622 coincides with the SOL where complete oxidation of $\mathrm{Ni}^{2+}$ is expected (at $x=0.66$ and $x=0.8$, respectively), thus evidencing that nickel oxidation occurs as a step-wise $\mathrm{Ni}^{2+} / \mathrm{Ni}^{3+}<_{-}>\mathrm{Ni}^{3+} / \mathrm{Ni}^{4+}$ process. All NCMs develop a pair of bands around 480 and $560 \mathrm{~cm}^{-1}$ upon delithiation that reach maximum intensity when lattice oxygen has been observed to escape the structure. The trends evidence the participation of oxygen in the charge-compensation process at low SOL. Based on the volume of released oxygen gas some authors argued that oxygen oxidation only occurs at the material's surface ${ }^{17}$ however, as the Raman laser penetrates hundreds of nanometers into the probed particles, our results indicate that oxygen redox is also a bulk phenomenon. Supporting evidence of bulk oxygen oxidation has been recently provided by X-ray absorption spectroscopy. ${ }^{76,77}$ On delithiation of NCM811 oxygen oxidation precedes the redox peak at $4.19 \mathrm{~V}$ associated with the rapid c-axis lattice contraction, possibly indicating that there is a narrow SOL window $(\Delta x \sim 0.05)$ where oxygen oxidation can contribute to the charge-compensation process without provoking the mechanical collapse of the lattice.

The occurrence of the redox peak at $3.74 \mathrm{~V}$ coincides with the appearance and growth of a band around $600 \mathrm{~cm}^{-1}$ in the operando Raman spectra of NCM622 and NCM811 (also previously observed on Mn-free NCA). The band, assigned to a short-ranged Li-ordering, evidences that the redox peak common for Ni-containing layered oxides ${ }^{43}$ (including NCM111, Figure 1a) originates from a configurational phase transition. The universality of the process supports a theoretically-predicted interaction between the electronic configuration of $\mathrm{Ni}$ and the occurrence of the lithium-ordering transition, ${ }^{11}$ and also suggests that such transitions can be potentially tuned by controlling the electronic structure of the oxide.

The demonstrated sensitivity of Raman spectroscopy to cycling-dependent processes in NCMs provides new opportunities for further monitoring degradation pathways and 
developing strategies for improving the electrochemical performance of the active material, e.g., by dopings and/or coatings.

\section{References}

1. Blomgren, G. E. The development and future of lithium ion batteries. J. Electrochem. Soc. 164, A5019-A5025 (2017).

2. Andre, D. et al. Future generations of cathode materials: an automotive industry perspective. J. Mater. Chem. A 3, 6709-6732 (2015).

3. He, P., Yu, H., Li, D. \& Zhou, H. Layered lithium transition metal oxide cathodes towards high energy lithium-ion batteries. $J$. Mater. Chem. 22, 3680-3695 (2012).

4. Myung, S. T. et al. Nickel-Rich Layered Cathode Materials for Automotive Lithium-Ion Batteries: Achievements and Perspectives. ACS Energy Lett. 2, 196-223 (2017).

5. Van der Ven, A., Aydinol, M., Ceder, G., Kresse, G. \& Hafner, J. First-principles investigation of phase stability in LixCoO2. Phys. Rev. B 58, 2975-2987 (1998).

6. Arroyo y de Dompablo, M. E., Van der Ven, A. \& Ceder, G. First-principles calculations of lithium ordering and phase stability on $<$ math display="inline" $><$ mrow $><$ msub $><$ mrow $>$ $<$ mi mathvariant="normal" $>$ Li $</$ mi $>\quad<$ mrow $>\quad<$ mrow $>$ $<$ mi $>$ x $</$ mi $><$ mrow $><$ /msub $><$ mrow $><$ mrow $><$ msub $>$ $<$ mrow $><$ mi mathvariant $=$ "normal" $>\mathrm{NiO}</ \mathrm{mi}>$. Phys. Rev. B 66, 064112 (2002).

7. Van der Ven, A., Bhattacharya, J. \& Belak, A. A. Understanding Li Diffusion in Li-Intercalation Compounds. Acc. Chem. Res. 46, 1216-1225 (2013).

8. Kang, K., Meng, Y. S., Breger, J., Grey, C. P. \& Ceder, G. Electrodes with High Power and High Capacity for Rechargeable Lithium Batteries. Science (80-. ). 311, 977-980 (2006).

9. Schipper, F. et al. Review-Recent Advances and Remaining Challenges for Lithium Ion Battery Cathodes. J. Electrochem. Soc. 164, A6220-A6228 (2017).

10. Mishra, S. K. \& Ceder, G. Structural stability of lithium manganese oxides. Phys. Rev. B 59, 6120-6130 (1999).

11. Dompablo, M. E. A. De, Marianetti, C., Ven, A. Van Der \& Ceder, G. Jahn-Teller mediated ordering in layered $\mathrm{Li}_{\mathrm{x}} \mathrm{MO}_{2}$ compounds. 63, 1-9 (2001).

12. Johannes, M. D., Swider-Lyons, K. \& Love, C. T. Oxygen character in the density of states as an indicator of the stability of Li-ion battery cathode materials. Solid State Ionics 286, 83-89 (2016).

13. Abraham, D. P. et al. Microscopy and Spectroscopy of Lithium Nickel Oxide-Based Particles Used in High Power Lithium-Ion Cells. J. Electrochem. Soc. 150, A1450 (2003).

14. Lin, F. et al. Surface reconstruction and chemical evolution of stoichiometric layered cathode materials for lithium-ion batteries. Nat. Commun. 5, 1688-1691 (2014).

15. Zhang, H. et al. Rock-Salt Growth-Induced (003) Cracking in a Layered Positive Electrode for Li-Ion Batteries. ACS Energy Lett. 2, 2607-2615 (2017).

16. Jung, R., Metzger, M., Maglia, F., Stinner, C. \& Gasteiger, H. A. Oxygen Release and Its Effect on the Cycling Stability of LiNi x Mn y Co z O 2 (NMC) Cathode Materials for Li-Ion Batteries. $J$. Electrochem. Soc. 164, A1361-A1377 (2017).

17. Streich, D. et al. Operando Monitoring of Early Ni-mediated Surface Reconstruction in Layered Lithiated Ni-Co-Mn Oxides. J. Phys. Chem. C 121, 13481-13486 (2017).

18. Jung, R., Metzger, M., Maglia, F., Stinner, C. \& Gasteiger, H. A. Chemical versus Electrochemical Electrolyte Oxidation on NMC111, NMC622, NMC811, LNMO, and Conductive Carbon. J. Phys. Chem. Lett. 8, 4820-4825 (2017)

19. Sun, H. \& Zhao, K. Electronic structure and comparative properties of $\mathrm{LiNi}_{\mathrm{x}} \mathrm{Mn}_{\mathrm{y}} \mathrm{Co}_{\mathrm{z}} \mathrm{O}_{2}$ cathode materials. J. Phys. Chem. C 121, 6002-6010 (2017).

20. Flores, E., Vonrüti, N., Novák, P., Aschauer, U. \& Berg, E. J. Elucidation of Li x Ni 0.8 Co 0.15 Al 0.05 O 2 Redox Chemistry by Operando Raman Spectroscopy. Chem. Mater. 30, 4694-4703 (2018).

21. Flores, E., Novák, P. \& Berg, E. J. In situ and Operando Raman Spectroscopy of Layered Transition Metal Oxides for Li-ion

Battery Cathodes. Front. Energy Res. 6, 1-16 (2018).

23
Baddour-Hadjean, R. \& Pereira-Ramos, J.-P. Raman microspectrometry applied to the study of electrode materials for lithium batteries. Chem. Rev. 110, 1278-1319 (2009).

Fateley, W. G., McDevitt, N. T. \& Bentley, F. F. Infrared and Raman selection rules for lattice vibrations: the correlation method. Appl. Spectrosc. 25, 155-173 (1971).

Gouadec, G. \& Colomban, P. Raman Spectroscopy of nanomaterials: How spectra relate to disorder, particle size and mechanical properties. Prog. Cryst. Growth Charact. Mater. 53, 1-56 (2007).

Boulet-Roblin, L. et al. Versatile approach combining theoretical and experimental aspects of raman spectroscopy to investigate battery materials: the case of the $\mathrm{LiNi}_{0.5} \mathrm{Mn}_{1.5} \mathrm{O}_{4}$ spinel. $J$. Phys. Chem. C 120, 16377-16382 (2016).

Noda, I., Dowrey, A. E., Marcott, C., Story, G. M. \& Ozaki, Y. Generalized Two-Dimensional Correlation Spectroscopy. Appl. Spectrosc. 54, 236A-248A (2000).

Noda, I. Techniques of two-dimensional (2D) correlation spectroscopy useful in life science research 1. Biomed. Spectrosc. Imaging 4, 109-127 (2015).

Felten, J. et al. Vibrational spectroscopic image analysis of biological material using multivariate curve resolutionalternating least squares (MCR-ALS). Nat. Protoc. 10, 217-240 (2015).

Kresse, G. \& Hafner, J. Ab initio molecular-dynamics simulation of the liquid-metal-amorphous-semiconductor transition in germanium. Phys. Rev. B 49, 14251-14269 (1994).

Kresse, G. \& Furthmüller, J. Efficiency of ab initio total energy calculations for metals and semiconductors using a plane-wave basis set. Comput. Mater. Sci. 6, 15-50 (1996).

Kresse, G. \& Hafner, J. Ab initio molecular dynamics for liquid metals. Phys. Rev. B 47, 558-561 (1993).

Perdew, J. P., Burke, K. \& Ernzerhof, M. Generalized gradient approximation made simple. Phys. Rev. Lett. 77, 3865-3868 (1996).

Blöchl, P. E. Projector augmented-wave method. Phys. Rev. B 50, 17953-17979 (1994).

Joubert, D. From ultrasoft pseudopotentials to the projector augmented-wave method. Phys. Rev. B - Condens. Matter Mater. Phys. 59, 1758-1775 (1999).

Anisimov, V. I., Zaanen, J. \& Andersen, O. K. Band theory and Mott insulators: Hubbard U instead of Stoner I. Phys. Rev. B $\mathbf{4 4}$ 943-954 (1991).

Dudarev, S. L., Botton, G. A., Savrasov, S. Y., Humphreys, C. J. $\&$ Sutton, A. P. Electron-energy-loss spectra and the structural stability of nickel oxide: An LSDA+U study. Phys. Rev. B 57, 1505-1509 (1998)

Hoang, K. \& Johannes, M. D. Defect Physics and Chemistry in Layered Mixed Transition Metal Oxide Cathode Materials: (Ni,Co,Mn) vs (Ni,Co,Al). Chem. Mater. 28, 1325-1334 (2016).

Togo, A., Oba, F. \& Tanaka, I. First-principles calculations of the ferroelastic transition between rutile-type and $\mathrm{CaCl} 2$-type $\mathrm{SiO} 2$ at high pressures. Phys. Rev. B - Condens. Matter Mater. Phys. 78, 1-9 (2008).

Fonari, A. \& Stauffer, S. vasp raman.py. (https://github.com/raman-sc/VASP/, 2013).

Kondrakov, A. O. et al. Charge-Transfer-Induced Lattice Collapse in Ni-Rich NCM Cathode Materials during Delithiation. J. Phys. Chem. C 121, 24381-24388 (2017).

Noh, H. J., Youn, S., Yoon, C. S. \& Sun, Y. K. Comparison of the structural and electrochemical properties of layered $\mathrm{Li}[\mathrm{NixCoyMnz}] \mathrm{O} 2(\mathrm{x}=1 / 3,0.5,0.6,0.7,0.8$ and 0.85$)$ cathode material for lithium-ion batteries. J. Power Sources 233, 121-130 (2013).

Ryu, H. H., Park, K. J., Yoon, C. S. \& Sun, Y. K. Capacity fading of ni-rich li[NixCoyMn1-x-y]O2 $(0.6 \leq \mathrm{x} \leq 0.95)$ Cathodes for High-Energy-Density Lithium-Ion Batteries: Bulk or Surface Degradation? Chem. Mater. 30, 1155-1163 (2018).

De Biasi, L. et al. Between Scylla and Charybdis: Balancing among Structural Stability and Energy Density of Layered NCM Cathode Materials for Advanced Lithium-Ion Batteries. J. Phys. Chem. C 121, 26163-26171 (2017).

Sun, H.-H. \& Manthiram, A. Impact of Microcrack Generation 
and Surface Degradation on a Nickel-Rich Layered Li[Ni 0.9 Co $0.05 \mathrm{Mn} 0.05$ ]O 2 Cathode for Lithium-Ion Batteries. Chem. Mater. 29, 8486-8493 (2017).

45. Tsai, Y. W. et al. In-situ X-ray absorption spectroscopic study on variation of electronic transitions and local structure of $\mathrm{LiNi}_{1 / 3} \mathrm{Co}_{1 / 3} \mathrm{Mn}_{1 / 3} \mathrm{O}_{2}$ cathode material during electrochemical cycling. Chem. Mater. 17, 3191-3199 (2005).

Qiao, R. et al. Transition-metal redox evolution in LiNi0.5Mn0.3Co0.2O2electrodes at high potentials. J. Power Sources 360, 294-300 (2017).

47. Eom, J., Kim, M. G. \& Cho, J. Storage Characteristics of LiNi[sub 0.8 ] Co[sub $0.1+x$ ] Mn[sub $0.1-x$ ] O[sub 2] $(x=0,0.03$, and 0.06) Cathode Materials for Lithium Batteries. $J$. Electrochem. Soc. 155, A239 (2008).

48. Jung, R. et al. Effect of Ambient Storage on the Degradation of Ni-Rich Positive Electrode Materials (NMC811) for Li-Ion Batteries. J. Electrochem. Soc. 165, A132-A141 (2018).

49. Liu, T., Garsuch, A., Chesneau, F. \& Lucht, B. L. Surface phenomena of high energy $\mathrm{Li}(\mathrm{Ni} 1 / 3 \mathrm{Co} 1 / 3 \mathrm{Mn} 1 / 3) \mathrm{O} 2 /$ graphite cells at high temperature and high cutoff voltages. J. Power Sources 269, 920-926 (2014).

50. Gallus, D. R. et al. The influence of different conducting salts on the metal dissolution and capacity fading of $\mathrm{NCM}$ cathode material. Electrochim. Acta 134, 393-398 (2014).

51. Ceder, G. \& Van der Ven, A. Phase diagrams of lithium transition metal oxides: investigations from first principles. Electrochim. Acta 45, 131-150 (1999).

52. Li, W., Reimers, J. N. \& Dahn, J. R. In situ X-ray diffraction and electrochemical studies of Li1-xNiO2. Solid State Ionics 67, 123130 (1993).

53. Reimers, J. N. \& Dahn, J. R. Electrochemical and In Situ X-Ray Diffraction Studies of Lithium Intercalation in $\mathrm{Li}[\mathrm{sub} \mathrm{x}] \mathrm{CoO}$ [sub 2]. J. Electrochem. Soc. 139, 2091 (1992).

54. Yin, S.-C., Rho, Y.-H., Swainson, I. \& Nazar, L. F. X-ray/neutron diffraction and electrochemical studies of lithium de/reintercalation in $\mathrm{Li}_{1-\mathrm{x}} \mathrm{Co}_{1 / 3} \mathrm{Ni}_{1 / 3} \mathrm{Mn}_{1 / 3} \mathrm{O}_{2}(\mathrm{x}=0 \rightarrow 1)$. Chem. Mater. 18, 1901-1910 (2006).

55. Ryu, H.-H., Park, G.-T., Yoon, C. S. \& Sun, Y.-K. Microstructural Degradation of Ni-Rich Li[Ni x Co y Mn $1-\mathrm{x}-\mathrm{y}$ ]O 2 Cathodes During Accelerated Calendar Aging. Small 14, 1803179 (2018)

56. Aroyo, M. I. et al. Bilbao Crystallographic Server: I. Databases and crystallographic computing programs. Zeitschrift fur Krist. 221, 15-27 (2006).

57. Reimers, J. N. Electrochemical and in situ X-ray diffraction studies of lithium intercalation in $\mathrm{Li}_{x} \mathrm{CoO}_{2}$. J. Electrochem. Soc. 139, 2091 (1992).

58. Julien, C. Local cationic environment in lithium nickel-cobalt oxides used as cathode materials for lithium batteries. Solid State Ionics 136-137, 887-896 (2000).

59. Rousseau, D. L., Bauman, R. P. \& Porto, S. P. S. Normal mode determination in crystals. J. Raman Spectrosc. 10, 253-290 (1981).

60. Kerlau, M., Marcinek, M., Srinivasan, V. \& Kostecki, R. M. Studies of local degradation phenomena in composite cathodes for lithium-ion batteries. Electrochim. Acta 52, 5422-5429 (2007).

61. Lanz, P., Villevieille, C. \& Novák, P. Ex situ and in situ Raman microscopic investigation of the differences between stoichiometric $\mathrm{LiMO}_{2}$ and high-energy $\mathrm{xLi}_{2} \mathrm{MnO}_{3} \cdot(1-\mathrm{x}) \mathrm{LiMO}_{2}$ $(\mathrm{M}=\mathrm{Ni}, \mathrm{Co}, \mathrm{Mn})$. Electrochim. Acta 130, 206-212 (2014).

62. Saavedra-Arias, J. J., Thomas, R., Karan, N. K., Ishikawa, Y. \& Katiyar, R. S. First principles computations studies: structural and electrochemical behavior of layered cathode materials. in ECS Transactions 16, 9-18 (ECS, 2009).

63. Liang, C. et al. Unraveling the Origin of Instability in Ni-Rich LiNi1-2xCoxMnxO2(NCM) Cathode Materials. J. Phys. Chem. C 120, 6383-6393 (2016).

64. Lee, K.-S., Myung, S.-T., Amine, K., Yashiro, H. \& Sun, Y.-K. Structural and Electrochemical Properties of Layered Li[Ni[sub $\left.1-2 \mathrm{x}] \mathrm{Co}\left[\begin{array}{ll}\mathrm{sub} & \mathrm{x}\end{array}\right] \mathrm{Mn}[\mathrm{sub} \quad \mathrm{x}]\right] \mathrm{O}\left[\begin{array}{ll}\mathrm{sub} & 2\end{array}\right] \quad(\mathrm{x}=0.1-0.3)$ Positive Electrode Materials for Li-Ion Batteries. J. Electrochem. Soc. 154, A971 (2007).

65. Yabuuchi, N., Koyama, Y., Nakayama, N. \& Ohzuku, T. SolidState Chemistry and Electrochemistry of LiCo[sub 1/3]Ni[sub
1/3] Mn[sub 1/3] O[sub 2] for Advanced Lithium-Ion Batteries. $J$. Electrochem. Soc. 152, A1434 (2005).

66. Marianetti, C. A., Morgan, D. \& Ceder, G. First-principles investigation of the cooperative Jahn-Teller effect for octahedrally coordinated transition-metal ions. Phys. Rev. B 63 224304 (2001).

67. Bersuker, I. Structure and Properties of Transition Metal Compounds. (John Wiley \& Sons, Inc., 2010)

68. Wood, E. A. Vocabulary of Surface Crystallography. Cit. J. Appl. Phys. 35, 1306 (1964).

69. Cahill, L. S. et al. 6 Li NMR Studies of Cation Disorder and Transition Metal Ordering in Li[Ni 1/3 Mn 1/3 Co 1/3 ]O 2 Using Ultrafast Magic Angle Spinning. (2005). doi:10.1021/cm0508773

70. Zeng, D., Cabana, J., Bréger, J., Yoon, W. \& Grey, C. P. Cation ordering in $\mathrm{Li}\left[\mathrm{Ni}_{\mathrm{x}} \mathrm{Mn}_{\mathrm{x}} \mathrm{Co}_{(1-2 \mathrm{x})}\right] \mathrm{O}_{2}$-layered cathode materials: a nuclear magnetic resonance (NMR), pair distribution function, $\mathrm{X}$ ray absorption spectroscopy, and electrochemical study. Chem. Mater. 19, 6277-6289 (2007).

71. WHITFIELD, P., DAVIDSON, I., CRANSWICK, L., SWAINSON, I. \& STEPHENS, P. Investigation of possible superstructure and cation disorder in the lithium battery cathode material LiMnNiCoO using neutron and anomalous dispersion powder diffraction. Solid State Ionics 176, 463-471 (2005).

72. Liu, W. et al. Nickel-rich layered lithium transition-metal oxide for high-energy lithium-ion batteries. Angew. Chemie Int. Ed. 54, 4440-4457 (2015).

73. Shannon, R. D. Revised effective ionic radii and systematic studies of interatomic distances in halides and chalcogenides. Acta Crystallogr. Sect. A A32, 751-767 (1976).

74. Dixit, M., Markovsky, B., Schipper, F., Aurbach, D. \& Major, D. $\mathrm{T}$. Origin of structural degradation during cycling and low thermal stability of Ni-rich layered transition metal-based electrode materials. J. Phys. Chem. C 121, 22628-22636 (2017).

75. Nakai, I., Takahashi, K., Shiraishi, Y., Nakagome, T. \& Nishikawa, F. Study of the Jahn-Teller distortion in $\mathrm{LiNiO}_{2}$, a cathode material in a rechargeable lithium battery, by in situ Xray absorption fine structure analysis. J. Solid State Chem. 140, 145-148 (1998)

76. Kleiner, $\mathrm{K}$. et al. Unraveling the degradation process of $\mathrm{LiNi}_{0.8} \mathrm{Co}_{0.15} \mathrm{Al}_{0.05} \mathrm{O}_{2}$ electrodes in commercial lithium ion batteries by electronic structure investigations. ACS Appl. Mater. Interfaces 7, 19589-19600 (2015).

77. Tian, C. et al. Depth-Dependent Redox Behavior of LiNi 0.6 Mn ${ }_{0.2} \mathrm{Co}_{0.2} \mathrm{O}_{2}$. J. Electrochem. Soc. 165, A696-A704 (2018). 